\title{
Comparative analysis of asexual and sexual stage Plasmodium falciparum development in different red blood cell types
}

\author{
Linda E. Amoah ${ }^{1,2^{*}} \mathbb{D}$, Festus K. Acquah ${ }^{1,2}$, Prince B. Nyarko², Elizabeth Cudjoe ${ }^{1}$, Dickson Donu', \\ Ruth Ayanful-Torgby' ${ }^{1}$ Fredericka Sey ${ }^{3}$, Kim C. Williamson $^{4}$ and Gordon A. Awandare ${ }^{2}$
}

\begin{abstract}
Background: Red blood cell (RBC) polymorphisms are suggested to influence the course of Plasmodium falciparum malaria. Whereas some variants have been found to be protective, others have been found to enhance parasite development. This study evaluated the effect of variant haemoglobin $(\mathrm{Hb})$ and $\mathrm{ABO}$ blood groups on P. falciparum merozoite invasion, multiplication rates as well as gametocyte development.

Methods: Approximately $2.5 \mathrm{~mL}$ of venous blood was collected from each participant. Flow cytometry was used to determine the in vitro merozoite invasion rates of NF54 parasites into the blood of 66 non-parasitaemic individuals with variant $\mathrm{Hb}$ genotypes ( $\mathrm{HbSS}, \mathrm{HbSC})$ and blood groups $(\mathrm{A}, \mathrm{B}, \mathrm{O})$, which were then compared with invasion into HbAA blood. The ex vivo asexual parasite multiplication and gametocyte production rates of parasites from 79 uncomplicated malaria patients with varying $\mathrm{Hb}$ genotypes ( $\mathrm{HbAS}, \mathrm{HbAC}$ and $\mathrm{HbAA}$ ) were also estimated using microscopy.
\end{abstract}

Results: Merozoite invasion rates were significantly reduced by about 50\% in RBCs containing HbSS and HbSC relative to $\mathrm{HbAA}$ cells. The presence of blood group $\mathrm{O}$ and $\mathrm{B}$ reduced the invasion rates of $\mathrm{HbSS}$ by about $50 \%$ and $60 \%$, respectively, relative to $\mathrm{HbSC}$ but the presence of blood group A removed the inhibitory effect of $\mathrm{HbSS}$. The initial parasite densities in uncomplicated malaria patients with $\mathrm{Hb}$ genotypes $\mathrm{HbAS}$ and $\mathrm{HbAC}$ cells were similar but significantly lower than those with genotype HbAA. The ex vivo parasite multiplication rate, gametocytaemia and gametocyte conversion rates followed a similar trend but did not reach statistical significance ( $p>0.05)$.

Conclusions: Parasite invasion rate into erythrocytes is dependent on both erythrocyte blood group antigen and haemoglobin genotype as blood group $\mathrm{O}$ and $\mathrm{B}$ provided protection via reduced merozoite invasion in RBCs containing $\mathrm{HbSS}$ relative to HbSC. Regardless of haemoglobin type, greater than $70 \%$ malaria patients had circulating ring stage parasites that differentiated into stage II gametocytes in 4 days.

Keywords: Haemoglobinopathies, Malaria, Gametocyte, Merozoite

*Correspondence: lamoah@noguchi.ug.edu.gh

1 Department of Immunology, Noguchi Memorial Institute for Medical Research, University of Ghana, Accra, Ghana

Full list of author information is available at the end of the article

\section{Background}

The selective force of Plasmodium infections in malariaendemic countries has resulted in human resistance to the disease. The main genetic determinants of this resistance include glucose-6-phosphate dehydrogenase (G6PD) deficiency [1], thalassemias [2], haemoglobinopathies (beta haemoglobin gene variants [3-5]) as well as blood group 'O' [6]. 
The beta haemoglobin variants that offer protection against malaria include $\mathrm{HbC}$, which is an abnormality in the $\beta$-globin subunit of the haemoglobin gene causing the glutamic acid residue at position 6 to be replaced with a lysine residue [4, 5], and $\mathrm{HbS}[3]$, in which the same glutamic acid is substituted with valine. Individuals who are homozygous for $\mathrm{HbCC}$ have been found to be strongly protected against severe malaria, while heterozygous $\mathrm{HbAC}$ individuals were found to be only mildly protected [7]. With HbS, the homozygous state is associated with severe complications, which usually results in death [8]. However, the heterozygous state, HbAS is associated with protection from severe as well as uncomplicated malaria $[7,9,10]$ and hospitalization due to malaria [11].

Although a number of studies have reported of the protective effects of variant $\mathrm{HbC}$ and $\mathrm{HbS}$, only a few have provided mechanisms that justify the associations [12]. While mechanisms such as RBC structural changes and its impact on disease progression and pathology have been proven [13-15], some other mechanisms including the impaired trafficking of PfEMP1 on the infected RBC surface [16] and activation of heme oxygenase by $\mathrm{HbS}$ [17] have been reported but not validated [18].

All ages of human red blood cells can be invaded by Plasmodium falciparum merozoites [19]. The invasion process has been studied directly using live microscopy $[20,21]$ and indirectly by determining the number of newly infected RBCs with ring-stage parasites using light or fluorescent microscopy [22, 23] and flow cytometrybase assays [24-27]. Successful invasion of RBCs by a merozoite results in the initiation of the erythrocytic life cycle of the parasite, where both asexual and sexual stage (gametocytes) parasites develop [28-30]. Merozoite invasion rates have been positively correlated with haematocrit [31], while red blood cell (RBC) polymorphisms including surface antigen diversity have been found to reduce the efficiency of merozoite invasion [19, 32].

Plasmodium falciparum gametocytes, which develop through five stages within the RBC, the early stages (stage I-II) and the late stages (stage III-V) [33] are derived from the asexual parasite. Only mature stage V gametocytes circulate in peripheral blood, while the immature stages are sequestered primarily in the bone marrow [34]. Stage V gametocyte density has been found to be associated with anaemia [35] and the presence of RBCs containing $\mathrm{HbC}$ and $\mathrm{HbS}$ [36]. However, a recent work that quantified gametocyte committed ring stage parasites in uncomplicated malaria patients indicated that gametocytaemia was associated positively with parasitaemia and negatively with fever but not haemoglobin levels [37]. Haemoglobin genotype was however not assessed.

Asexual parasite replication usually results in the presentation of signs and symptoms of malaria in the infected hosts (symptomatic infections). However, other infections can remain asymptomatic. Similarly, sexual stage parasite (gametocyte) development within the host's erythrocyte is not associated with disease pathology. Asymptomatic parasite carriage (both asexual and gametocyte) is a huge burden to malaria control as the host remains afebrile and does not seek treatment. As global efforts move from malaria control towards elimination and global eradication, identifying gametocyte carriage and factors that influence malaria transmission has become a necessity [38]. With haemoglobin variants suggested to enhance malaria transmission [36], malaria control strategies will benefit from knowledge on how these variants affect parasite growth and gametocyte carriage.

To determine the contribution of host RBC polymorphisms on the establishment and transmission of malaria, this study explored $P$. falciparum merozoite invasion and multiplication rates, as well as gametocyte development in RBCs containing variant $\beta$-globin $(\mathrm{Hb})$ genotypes and ABO blood groups. Significant differences in merozoite invasion were observed between the variant $\mathrm{Hb}$ genotypes as well as among the three major blood groups. Parasite multiplication rates and gametocyte production rates were however not significantly different among the variant $\mathrm{Hb}$ genotypes tested.

\section{Methods}

\section{Ethical statement}

The Institutional Review Board of the Noguchi Memorial Institute for Medical Research (NMIMR), University of Ghana provided approval for the study (protocols \# 085/12-13 and 024/14-15). Prior to enrollment, all study participants, including men, women and children were educated on the purpose of the study. Afterwards, a written informed consent, assent and parental consent were obtained from the participants and/or their parents (guardians).

\section{Study site and population}

Samples for the study were obtained from two cohorts; one comprised of a group of children with uncomplicated malaria (UM) and the other comprised of afebrile participants with haemoglobin HbSS or HbSC. A total of 79 children aged between 6 and 15 years with uncomplicated malaria attending the Ewim Health Centre were recruited between July 2015 and August 2017. A second set of 66 afebrile participants aged between 13 and 47 years, attending routine check-up at the Sickle Cell Clinic between June 2016 to April 2017 were also recruited into a cross sectional study. An additional set of 4 healthy volunteers aged between 25 and 35 years with HbAA RBCs were recruited from the NMIMR to supply 
blood for the in vitro parasite cultures and also serve as controls for the merozoite invasion assay.

The Sickle Cell Clinic at the Ghana Institute of Clinical Genetics, Korle-Bu is situated within the Accra metropolis of the Greater Accra Region of Ghana. The Ewim polyclinic is in the Cape Coast metropolis of the Central Region of Ghana.

\section{Sample acquisition and processing}

Each study participant donated three drops of fingerpricked blood $(\sim 150 \mu \mathrm{L})$, which was used to prepare thick and thin blood smears, spot an HRP2-RDT and filter paper blood blots (Whatman ${ }^{\circledR} \# 3$, GE Healthcare, USA) for gDNA extraction and $\mathrm{ABO}$ blood grouping. Additionally, $2.5 \mathrm{~mL}$ of venous blood was collected from each participant into acid citrate dextrose (ACD) tubes for use in parasite cultures. All persons attending the various health facilities had their axillary temperature measured using a handheld digital thermometer.

Venous blood from the afebrile volunteers was separated by centrifugation and the plasma stored at $-20^{\circ} \mathrm{C}$. The blood cell pellets were washed twice with 2 volumes of incomplete parasite medium (iCPM: RPMI 1640 supplemented with $25 \mathrm{mM}$ Hepes, $2 \mathrm{mM}$ L-glutamine, $25 \mathrm{mM} \mathrm{NaHCO}, 20 \mathrm{mM}$ glucose, $5 \mu \mathrm{g} / \mathrm{mL}$ gentamycin, $50 \mu \mathrm{g} / \mathrm{mL}$ of hypoxanthine). The pelleted RBCs were finally resuspended in an equal volume of complete parasite media (CPM: iCPM supplemented with 0.5\% Albumax II and 2\% of normal human serum) and kept at $4{ }^{\circ} \mathrm{C}$ for no longer than 3 days. The whole blood collected from the children with uncomplicated malaria was processed as described for the healthy volunteers above. However, these RBCs were re-suspended at 3\% haematocrit and used immediately to plate the ex vivo assay.

\section{ABO blood grouping}

ABO blood group phenotyping was performed based on the forward tile method adopted from Karl Landsteiner's haemagglutination protocol, using a commercially available kit (Accucare, Lab Care Diagnostics, India) according to manufacturer's instructions. Briefly, three separate small drops $(20 \mu \mathrm{L})$ of plasma from each individual were put on a glass slide. An equal volume of $(20 \mu \mathrm{L})$ of antiserum $\mathrm{A}, \mathrm{B}$ or $\mathrm{O}$ (from the test kit) was individually added to the blood spot and mixed immediately. Blood group was scored after visually observing the mixture for evidence of agglutination within $2 \mathrm{~min}$.

\section{Beta globin genotyping}

The haemoglobin variant genotyping was performed using the PCR-RFLP protocol previously described by Danquah et al. [39] to detect the homo- and heterozygous $\mathrm{A}, \mathrm{C}$ and $\mathrm{S}$ haemoglobin alleles. Amplification of a
$358 \mathrm{bp}$ region of the $\beta$ globin gene was done in a $30 \mu \mathrm{L}$ reaction volume using $200 \mathrm{nM}$ of SC1F (5'-AGGAGC AGGGAGGGCAGGA-3') and SC2R (5'-TCCAAGGGT AGACCACCAGC-3') oligonucleotide primers. The reaction contained $1 \times$ GC rich buffer, $200 \mathrm{nM} \mathrm{dNTP,} 150 \mathrm{nM}$ $\mathrm{MgCl}_{2}$, and $1 \mathrm{U}$ of One Taq DNA polymerase (Thermo Fisher Scientific, UK). The cycling conditions were; initial denaturation at $94{ }^{\circ} \mathrm{C}$ for $5 \mathrm{~min}$, followed by 35 cycles with $94{ }^{\circ} \mathrm{C}$ for $50 \mathrm{~s}, 64{ }^{\circ} \mathrm{C}$ for $50 \mathrm{~s}$ and $72{ }^{\circ} \mathrm{C}$ for $40 \mathrm{~s}$; with a final extension at $72{ }^{\circ} \mathrm{C}$ for $5 \mathrm{~min}$. The PCR products were subjected to digestion with $M n l I$ and DdeI restriction enzymes (New England BioLabs, UK) by incubating at $37^{\circ} \mathrm{C}$ for 30 min to detected $\mathrm{HbSC}$ and HbSS genotypes. All PCR-RFLP products were resolved on 3\% agarose gels and fragment sizes were used to determine the various $\mathrm{Hb}$ genotypes as previously described [39].

\section{In vitro parasite culture}

A continuous culture of the $P$. falciparum NF54 strain was maintained at $2 \%$ haematocrit in complete parasite media (CPM: RPMI 1640 supplemented with HEPES, L-glutamine, $\mathrm{NaHCO}_{3}$, glucose, gentamycin and Albumax II). Parasites were cultured in vitro using a modified version of the method by Trager and Jensen [40]. Briefly, parasites were maintained in $\mathrm{O}+$ red blood cells (RBCs), equilibrated in a blood gas environment of $94 \%$ nitrogen, $5 \% \mathrm{CO}_{2}, 1 \%$ oxygen (Air Liquide, Birmingham, UK) and maintained in an incubator at $37{ }^{\circ} \mathrm{C}$ with daily media change and periodic supplementation of uninfected RBCs to maintain the parasitaemia below 5\% until the cultures had expanded enough to be used for an assay. At $5 \%$ parasitaemia, the culture was synchronized with $5 \%$ sorbitol [41] and then allowed to expand with daily media change and thin smear preparation to monitor parasite growth and development. The culture was harvested at high $(\sim 15 \%)$ schizont population and used for the invasion assays.

\section{Ex vivo gametocyte and asexual parasite culture of samples from children with uncomplicated malaria}

An aliquot $(50 \mu \mathrm{L})$ of packed washed RBCs from the children with uncomplicated malaria (samples from the cohort with RBCs containing $\mathrm{HbAA}, \mathrm{HbAS}$ and $\mathrm{HbAC}$ ) were maintained in culture at $37^{\circ} \mathrm{C}$ in duplicate wells of a 12-well plate using CPM. For the gametocyte culture, the CPM in each well was supplemented with $50 \mathrm{mM}$ of $\mathrm{N}$-acetyl glucosamine (NAG) to prevent the expansion of the asexual parasite population and subsequent production of culture-induced gametocytes [37].

An asexual parasite culture was set up identical to that described for the gametocyte cultures. However, the media was not supplemented with NAG. This set up was used to serve as a control for the gametocyte assay and 
also to determine the ex vivo asexual parasite multiplication rate. The plates were placed in a Modular ${ }^{\circledR}$ incubating chamber, gassed for 6 min with mixed gas $(94 \%$ nitrogen, $5 \% \mathrm{CO}_{2}, 1 \%$ oxygen) and placed in an incubator set at $37{ }^{\circ} \mathrm{C}$. Parasite media was changed and thin smear prepared daily for 6 days, however, unlike the in vitro culture, no RBCs were added during the course of culturing.

\section{Evaluation of blood smears}

The thick and thin smears were processed according to standard protocol [42]. Briefly, the slides were air-dried, after which the thin smears were fixed in methanol. Thick and fixed thin smears were then stained with $10 \%$ Giemsa for $15 \mathrm{~min}$, after which they were air-dried and subsequently viewed under $100 \times$ oil immersion microscope [43]. Two independent microscopists read each slide.

\section{Evaluation of merozoite invasion into RBCs from afebrile individuals using flow cytometry}

Invasion assays were performed as previously described [27]. Briefly, to distinguish acceptor (target) cell (HbSS and $\mathrm{HbSC}$ ) from residual $\mathrm{RBCs}$ in parasite inoculum, acceptor cells were stained with a cytoplasmic fluorescent stain; 5-(and-6)-carboxyfluorescein diacetate succinimidyl ester (5(6) CFDA-SE; $20 \mu \mathrm{M}$; Invitrogen), prior to assay plating. Schizont stage parasites were mixed with the acceptor cells in a $1: 1$ ratio at $2 \%$ haematocrit in 96-well titre plates. All experiments were setup in duplicates. Assays were incubated overnight at $37^{\circ} \mathrm{C}$ in a blood gas environment of $94 \%$ nitrogen, $5 \% \mathrm{CO}_{2}, 1 \%$ oxygen (Air Liquide, Birmingham, UK). Assays were stained with $5 \mu$ M Hoechst (Hoechst 33342 Sigma Aldrich) after incubation to differentiate parasitized RBCs from uninfected ones. Invasion levels were determined by flow cytometry (LSR Fortessa X-20; BD) as described in other studies [25, 27]. Invasion levels in individual assays were determined by recording the proportion (in percentage) of RBCs that were dual positive for CFDA and Hoechst 33342. Invasion rates into RBCs containing $\mathrm{HbSS}$ and $\mathrm{HbSC}$ were then calculated by comparing their invasion levels to that of the control HbAA group, which was set at $100 \%$.

\section{Data analysis}

Parasite density was determined as the number of malaria parasites observed per 200 white blood cells on the thick smear, multiplied by 40 [44]. Parasitaemia measurements were determined as the percent of parasite-infected RBCs observed per 1000 RBCs on the thin smear. Parasite multiplication rate was defined as the fold-increase in asexual parasitaemia observed in cultures between D4 and D6. The production of early-stage (II) gametocytes on Day 4 was expressed as the percent of stage-specific infected RBCs counted per 20,000 RBCs [37].

BD FACSDIVA V8.0.1 was used to analyse the flow cytometry data. Invasion rate in the $\mathrm{RBC}$ variants was calculated as the percent of infected $\mathrm{RBC}$ contained in the patient blood (A) relative to that in the control blood (C) $[(\mathrm{A} / \mathrm{C}) * 100)]$.

GraphPad Prism 5.01 was used for all the statistical analysis, including descriptive statistics, $T$ test, KruskalWallis test and Dunn's Multiple Comparison test. Dunn's Multiple Comparison test was performed only when the Kruskal-Wallis test identified a significant difference. Parasite density in samples containing $\mathrm{HbAA}, \mathrm{HbAC}$ and HbAS on D0 was compared using Kruskal-Wallis test followed by Dunn's Multiple Comparison test. Parasite multiplication rates and gametocyte densities were compared using Kruskal-Wallis test. $p \leq 0.05$ was considered significant.

\section{Results}

\section{Demographics of study participants}

The study recruited 79 children aged between 6 and 15 years old with uncomplicated malaria, out of which $24 \%(19 / 79)$ had HbAC, 19\% (15/79) had HbAS and 60\% (45/79) had HbAA (Table 1). The HRP2-RDT positivity rate in the children with uncomplicated malaria was $100 \%$. The median parasite density in the HbAC and HbAS children were similar but the median parasite density for children with $\mathrm{HbAC}$ was significantly lower than those with the HbAA genotype (Table 1).

The second cohort comprised of 66 afebrile participants aged between 13 and 55 years. There were 53\% (35/66) of the afebrile study participants that had HbSS and another $47 \%(31 / 66)$ had HbSC (Table 2). The number of males

$\begin{aligned} & \text { Table } 1 \\
& \text { with uncomplicated malaria } \\
& \text { Demographic characteristics of }\end{aligned}$
\begin{tabular}{llll} 
UM & participants \\
\cline { 2 - 4 } & HbAenotype $(\mathbf{n}=\mathbf{4 5})$ & HbAC $(\mathbf{n}=\mathbf{1 9})$ & HbAS $(\mathbf{n}=\mathbf{1 5})$ \\
\hline Sex (F/M) & $20 / 22$ & $11 / 7$ & $5 / 10$ \\
Age (years) & & & \\
Min-max & $1-15$ & $1-15$ & $1-12$ \\
Median (IQR) & $6(3-8)$ & $5(4-9.5)$ & $7(5-8.25)$ \\
RDT (\%) & 100 & 100 & 100
\end{tabular}

Uncomplicated malaria-axillary temperature at or above $37.5^{\circ} \mathrm{C}$ and presence of malaria parasites by microscopy; afebrile-axillary temperature below $37.5^{\circ} \mathrm{C}$ and absence of malaria parasites by microscopy as well as negative by a malaria RDT

$F$ female, $M$ male, $B G$ blood group, $P D$ Parasite density measured as parasite, $p$ per microlitre; min minimum, max maximum, $I Q R$ interquartile range (25-75\%); $R D T$ HRP2 based malaria rapid diagnostic test positivity rate, Temp axillary temperature, $N D$ not done, $N P$ no parasite observed by microscopy 
Table 2 Demographic characteristics of the afebrile study participants

\begin{tabular}{lll}
\hline Afebrile & \multicolumn{2}{l}{ HB genotype } \\
\cline { 2 - 3 } & HbSS $(\mathbf{n}=\mathbf{3 5})$ & HbSC $(\mathbf{n = 3 1 )}$ \\
\hline Sex (F/M) & $16 / 19$ & $17 / 14$ \\
BG (O/A/B) & $24 / 7 / 4$ & $14 / 6 / 10$ \\
Age (years) & & \\
Min-max & $13-55$ & $14-47$ \\
Median (IQR) & $24(20-30)$ & $26(19-55)$ \\
\hline
\end{tabular}

Uncomplicated malaria-axillary temperature at or above $37.5^{\circ} \mathrm{C}$ and presence of malaria parasites by microscopy; afebrile-axillary temperature below $37.5^{\circ} \mathrm{C}$ and absence of malaria parasites by microscopy as well as negative by a malaria RDT

$F$ female, $M$ male, $B G$ blood group, $P D$ Parasite density measured as parasite, $p$ per microlitre; min minimum, max maximum, IQR interquartile range (25-75\%); $R D T$ HRP 2 based malaria rapid diagnostic test positivity rate, Temp axillary temperature, ND not done, NP no parasite observed by microscopy

and females in both cohorts was similar (Tables 1 and 2). The HRP2-RDT positivity rate and parasite densities of the afebrile participants were both 0 .

\section{Parasite density in children with uncomplicated malaria}

The asexual parasite densities in the samples collected from the uncomplicated malaria patients ranged from 2604 parasites $/ \mu \mathrm{L}$ to 249,960 parasites $/ \mu \mathrm{L}$, with a median (IQR) of $81,148 \pm 6648$ parasites $/ \mu \mathrm{L}$. The samples from participants with HbAA had the highest parasite densities, with a median (IQR) of 88,716 (43,740-164,775) parasites $/ \mu \mathrm{L}$ and the least was identified in samples with $\mathrm{HbAC}$, which had a median (IQR) parasite density of $24,615(17,043-32,804)$ parasites/ $\mu \mathrm{L}$ (Table 3). The parasite density in samples with HbAS was similar to that of $\mathrm{HbAC}$, which were both significantly lower than the parasite density in HbAA (Dunn's multiple comparison test, $\mathrm{p}<0.001$ for HbAA and HbAC and $\mathrm{p}<0.01$ for HbAA and HbAS (Fig. 1a).

\section{Parasite multiplication rate in RBCs from children with uncomplicated malaria}

The fold-increase of asexual parasite infected RBCs (multiplication rate) within each culture was measured in the control cultures (grown in the absence of NAG) of HbAA, HbAS and HbAC between D4 and D6. The asexual parasite multiplication rates ranged from a median (IQR) of $2.3(1.2-3.2)$ in HbAA, 1.55 (1.2-2.55) in HbAC and 1.35 (0.85-2.0) in HbAS cells (Fig. 1b, Additional file 1: Table S1). However, no statistical significant difference (Kruskal-Wallis test, $\mathrm{p}=0.056$ ) was observed among the multiplication rates between erythrocytes containing normal $\mathrm{HbAA}$ and the variant $\mathrm{Hb}$ (HbAS and HbAC) genotypes (Fig. 1b, Additional file 1: Table S1).

\section{Ex vivo gametocyte development in RBCs from children with uncomplicated malaria}

Gametocyte densities in the cultures from the symptomatic participants were determined after four (D4) days of continuous culture in the presence of NAG. The NAG in the media ensures that only gametocytes (stage II) produced from ring-stage parasites that were sexually committed at the time of the initial culture set up were determined. Gametocyte prevalence on D4 in all the three different RBC Hb types (HbAA, HbAS and $\mathrm{HbAC}$ ) were similar (Kruskal-Wallis test, $\mathrm{p}=0.166$ ), although cultures containing $\mathrm{HbAA}$ produced the

Table 3 Malariaometric indices of children with uncomplicated malaria

\begin{tabular}{|c|c|c|c|}
\hline & HbAA & $\mathrm{HbAC}$ & HbAS \\
\hline \multicolumn{4}{|l|}{ DO PD (p/ $\mu \mathrm{L})$} \\
\hline Count (n/N) & $100 \%(45 / 45)$ & $100 \%(19 / 19)$ & $100 \%(15 / 15)$ \\
\hline Median (IQR) & $88,716(43,740-164,775)$ & $24,615(17,043-32,804)$ & $23,540(9130-122,760)$ \\
\hline Min-max & $3910-249,960$ & $2604-226,830$ & $3621-248,116$ \\
\hline \multicolumn{4}{|l|}{ DO AS } \\
\hline Count $(\mathrm{n} / \mathrm{N})$ & $100 \%(45 / 45)$ & $100 \%(19 / 19)$ & $100 \%(15 / 15)$ \\
\hline Median (IQR) & $3.2(1.93-4.28)$ & $3.7(0.78-6.1)$ & $2.2(1.4-5.7)$ \\
\hline Min-max & $0.90-21$ & $0.78-13.30$ & $0.29-7.1$ \\
\hline \multicolumn{4}{|l|}{ D4 ES } \\
\hline Count $(n / N)$ & $95.5 \%(43 / 45)$ & $73.7 \%(14 / 19)$ & $80 \%(12 / 15)$ \\
\hline Median (IQR) & $0.08(0.02-0.25)$ & $0.05(0.01-0.12)$ & $0.03(0.01-0.10)$ \\
\hline Min-max & $0.004-1.39$ & $0.009-0.34$ & $0.004-0.486$ \\
\hline
\end{tabular}

Count stated as a percent followed by a fraction

$P D$ parasite density measured as parasites, $p$ per microlitre, $A S$ asexual $P$. falciparum parasites, ES early stage $P$. falciparum gametocytes, min minimum, max maximum, IQR interquartile range (25-75\%), $n$ number positive, $N$ total number present 

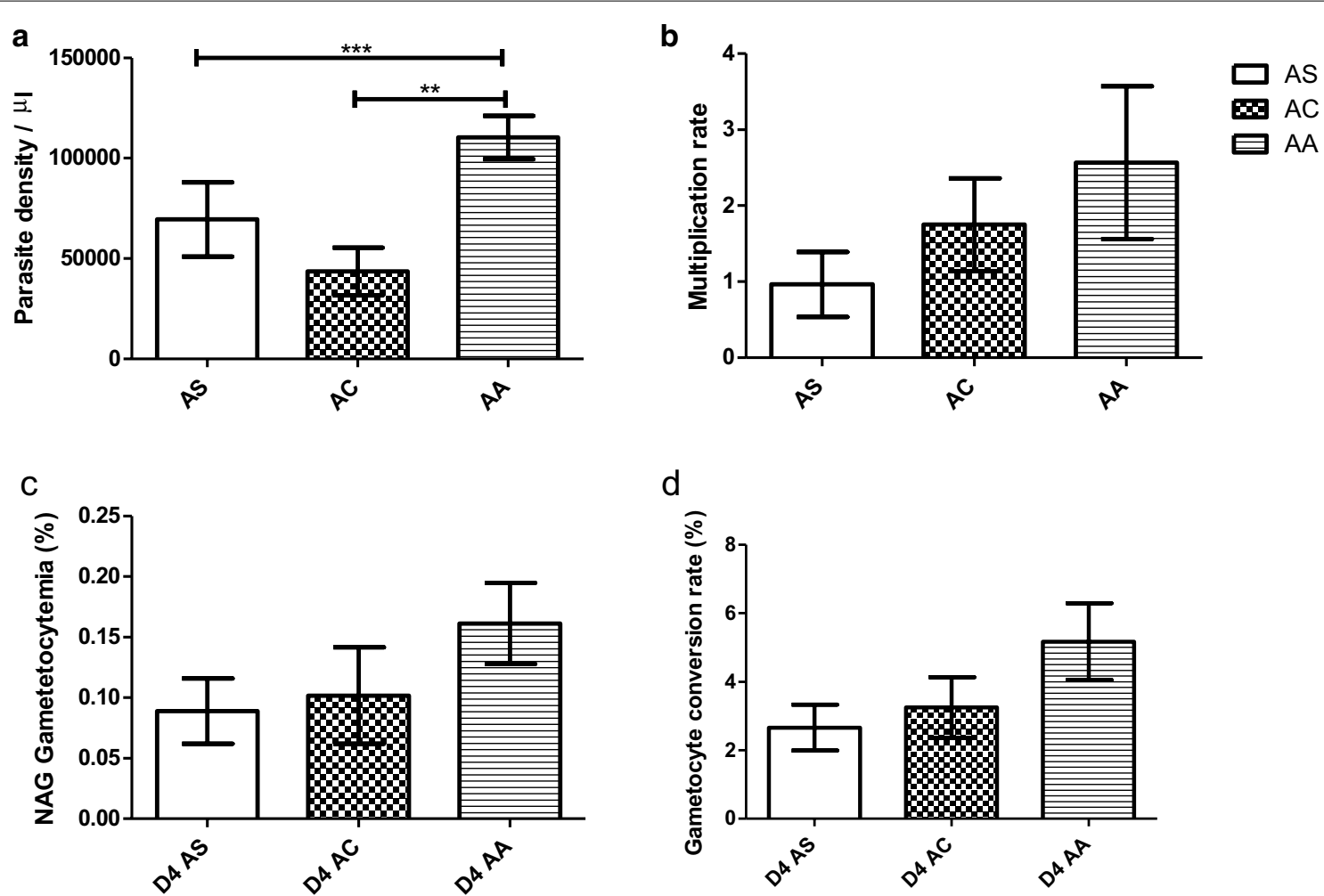

Fig. 1 Ex vivo parasite development in normal and variant $\mathrm{Hb}$ erythrocytes. a Parasite density on $\mathrm{D} 0, \mathbf{b}$ asexual parasite multiplication rates, $\mathbf{c}$ gametocytaemia on D4 and $\mathbf{d}$ gametocyte conversion rate. $\mathbf{a}$ Giemsa-stained thin blood smears prepared from $A S n=15, A C n=19$ and $A A$ $\mathrm{n}=45 \mathrm{D} 0$ samples were used to determined asexual parasitaemia. $\mathbf{b}$ The fold increase in asexual parasite densities in samples counted on D6 were compared to that counted in the same culture on D4 from $A S n=12, A C n=14$ and AA $n=40$ samples. $c$ Gametocyte densities in samples from $A S n=12, A C n=14$ and AA $n=43$ examined on D4. d D4 Gametocyte conversion rate, represented by the \% of the D0 ring stage parasites that develop into early stage (II) gametocytes by D4 from AS $n=12, A C n=14$ and AA $n=43$ samples. Parasite infected RBCs in the thin blood smears were counted against 1000 RBCs for asexual parasite density estimation and 20,000 RBCs for gametocyte densities

highest gametocytaemia (median, IQR) of, 0.08, 0.020.25 , while the lowest gametocytaemia of $0.03,0.01-0.10$ ) were found in HbAS cultures (Fig. 1c, Additional file 1: Table S1). The ratio between D4 gametocytemia and D0 parasitaemia, which represents the gametocyte conversion rate in $\mathrm{HbAA}, \mathrm{HbAS}$ and HbAC erythrocytes was also similar (Fig. 1d, Additional file 1: Table S1).

\section{Merozoite invasion rates in RBCs from afebrile individuals}

The median (IQR) invasion rates into RBCs containing HbSS and HbSC was 49.6\% (29.6-62.4) and 47.6\% (30.764.5) respectively, which were both significantly lower (Kruskal-Wallis test, $\mathrm{p}<0.0001$ ) than invasion into RBCs containing the normal HbAA genotype (median (IQR) of 99.5\% (98.6-101). Dunn's multiple comparison for HbSS vs HbAA and HbSC vs HbAA, $\mathrm{p}=0.001$ for both combinations) (Fig. 2a, Additional file 1). However, no significant difference was observed between the invasion rates into RBCs containing HbSC relative to HbSS (MannWhitney test, $p=0.977$ ).
The influence of varying blood group antigen on merozoite invasion rates into erythrocytes containing variant $\mathrm{Hb}$ genotypes, $\mathrm{HbSC}$ and $\mathrm{HbSS}$ combined was determined by sorting the invasion rate data for the variant RBCs according to blood group antigen. The median (IQR) invasion rates into the various blood groups were 60.35\% (39.8-66.5) in blood group A, 45.6\% (24.4-55.8) in blood group B and 47.1\% (28.8-64.8) in blood group O. Reduced merozoite invasion rates was observed in RBCs with blood groups B and O relative to blood group A, but the difference was only statistically significant between RBCs containing blood group B and A (Mann-Whitney test, $p=0.007$ ) (Fig. 2b, Additional file 1: Table S1).

To understand the individual contribution of blood group antigen to merozoite invasion into each of the groups of $\mathrm{Hb}$ variants (HbSC and HbSS separately), merozoite invasion for each blood group antigen was analysed separately for RBCs containing the HbSC and $\mathrm{HbSS}$. Merozoite invasion rates in HbSS containing red blood cells were similar in all of the ABO blood groups 

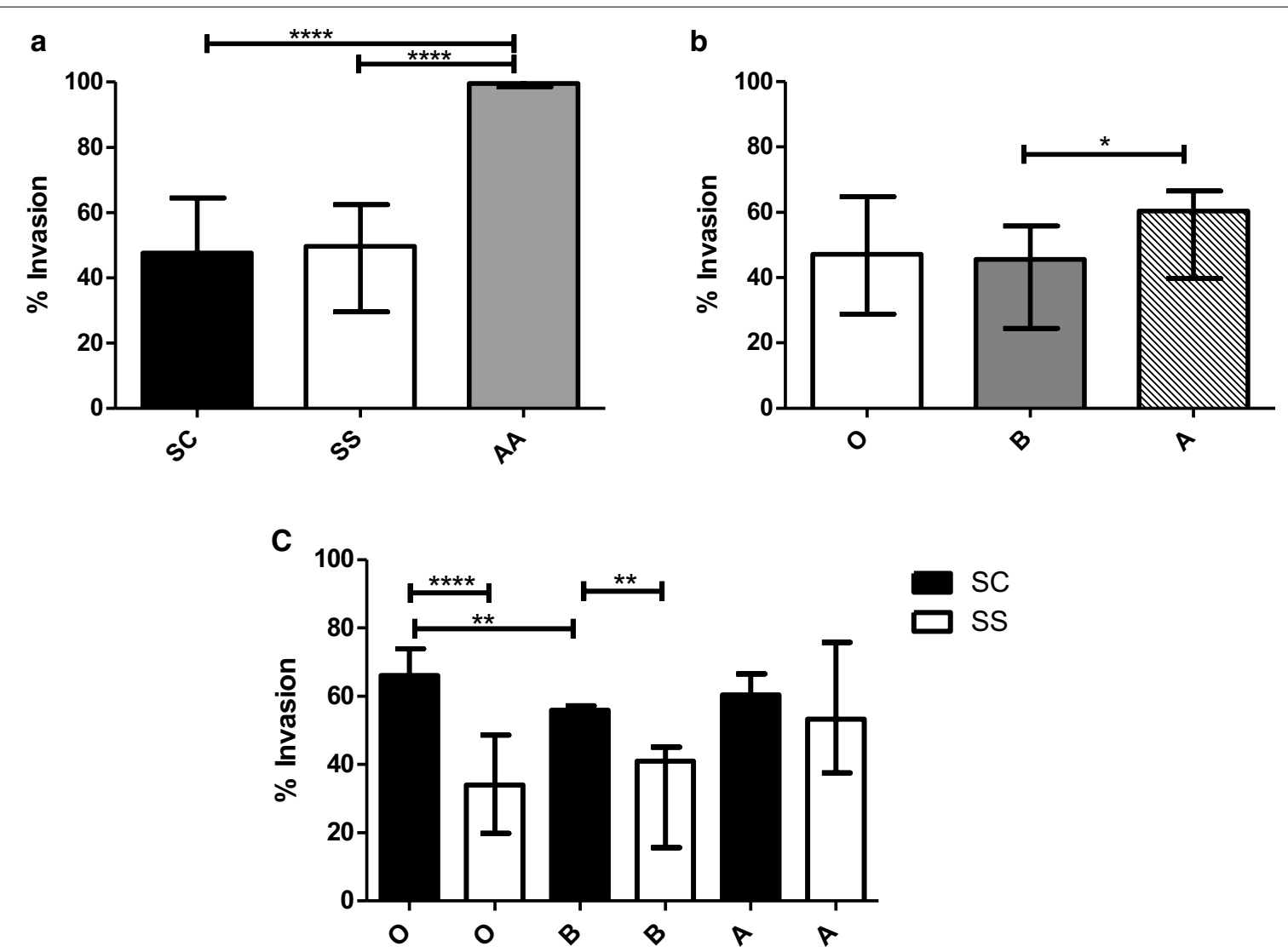

Fig. 2 Parasite invasion into normal and variant Hb erythrocytes. a Invasion of NF54 into erythrocytes with variant $(\mathrm{HbSS} n=35$ and $\mathrm{HbSC} n=31)$ and normal $(\mathrm{HbAA} n=4) \mathrm{Hb}$ genotypes. The assay was performed in duplicate. $\mathbf{b}$ Invasion rates in $\mathrm{HbSS}$ and $\mathrm{HbSC}$ samples with different blood groups, ' $O{ }^{\prime} n=38,{ }^{\prime} B^{\prime} n=14$ and $A{ }^{\prime} n=14$. c Invasion in HbSS ('O' $n=24,{ }^{\prime} A^{\prime} n=7,{ }^{\prime} B$ ' $\left.n=4\right)$ and HbSC ('O' $n=14,{ }^{\prime} A^{\prime} n=7,{ }^{\prime} B$ ' $\left.n=10\right)$ RBCs categorized according to blood group antigen. The data in the graphs represent the median and the error bars are the interquartile range

(Kruskal-Wallis test, $\mathrm{p}=0.054$ ). Among the $\mathrm{HbSC}$ containing RBCs, invasion rates were significantly different (Kruskal-Wallis test, $\mathrm{p}=0.002$ ), with invasion rates reduced in blood group B containing RBCs relative to the blood group O (Dunn's Multiple comparison test, $p<0.01$; Mann-Whitney test, $\mathrm{U}=58.5$, $\mathrm{p}=0.0003$ ) (Fig. 2c, Additional file 1: Table S1).

The median merozoite invasion rates in blood groups $\mathrm{O}$ and $\mathrm{B}$ were significantly higher in the $\mathrm{HbSC}$ group (66.06\% and $55.87 \%$ respectively) compared to blood groups $\mathrm{O}$ and $\mathrm{B}$ in the HbSS group (33.975 and 40.98\% respectively) (Mann-Whitney test, $U=174.5$, $\mathrm{p}<0.0001$ for blood group $\mathrm{O}$ and $\mathrm{U}=36.5, \mathrm{p}=0.919$ for blood group B). Merozoite invasion rates in blood group A RBCs with HbSC (60.35\%) was similar (Mann-Whitney test, $\mathrm{U}=77.5, \mathrm{p}<0.0001$ to those in HbSS group (53.32\%) (Fig. 2c, Additional file 1: Table S1).

\section{Discussion}

A number of studies have reported the sickle cell trait and haemoglobin $C$ to provide anti-disease immunity against both severe and uncomplicated malaria [3, 4, $10,45-48]$. A variety of mechanisms governing this phenomenon have been suggested. This study sought to characterize $\mathrm{RBC}$ invasion into RBCs containing a haemoglobinopathy (a variant $\mathrm{Hb}$ genotype) as well as compare gametocyte development and asexual parasite multiplication rates in RBCs containing HbAA and other haemoglobinopathies.

During a natural infection of uncomplicated malaria, children with $\mathrm{HbAC}$ and HbAS had significantly lower parasite densities than children with both HbAA, supporting previous reports which suggest a protective effect of haemoglobin variants $\mathrm{HbAC}$ and $\mathrm{HbAS}$ against high parasitaemia and severe disease [7, 49]. High parasite densities found in children with HbAA RBCs relative 
to RBCs with haemoglobinopathies has been previously reported $[9,45,47]$.

In contrast to asexual parasitaemia, $\mathrm{RBCs}$ with $\mathrm{HbC}$ and $\mathrm{HbS}$ genotypes have been reported to contain high numbers of stage V gametocytes [50]. However, none of the children in this study had microscopically detectable stage $\mathrm{V}$ gametocytes on D0, even though $>70 \%$ of the subjects had circulating ring stage parasites that differentiated into stage II-III by D4 of ex vivo culture. Few studies have also identified a large proportion of asymptomatic individuals $[51,52]$ as well as symptomatic malaria patients [53], irrespective of erythrocyte $\mathrm{Hb}$ genotype or blood group, to harbor submicroscopic densities of gametocytes. Although the initial D0 parasitaemias were lower in $\mathrm{HbAC}$ and $\mathrm{HbAS}$ subjects, no significant difference was observed between gametocyte densities in $\mathrm{HbAC}$ and HbAS RBCs relative to HbAA RBCs. This could be due to the presence of a higher initial number of gametocyte committed ring stage parasites in the $\mathrm{HbAC}$ and $\mathrm{HbAS}$ relative to the parasite population in the HbAA RBCs. However, this claim would need to be validated by real time PCR as gametocyte committed rings and asexual ring stage parasites are indistinguishable by microscopy [37].

The present study affirm observations that RBCs with $\mathrm{HbC}$ genotype have low invasion capabilities compared to normal HbAA genotype [54]. The data further suggests that the erythrocytic mechanism of the anti-disease protection is associated with invasion in the abnormal haemoglobin variants and not only due to the intraerythrocytic metabolic processes as previously suggested [3]. The 'O' blood group has been associated with protection against severe forms of malaria relative to non-' $\mathrm{O}$ ' blood groups because the glycoproteins of non-'O' blood groups serve as receptors for a number of biological processes associated with malaria, including merozoite invasion and rosetting in the parasite $[6,48]$. In this study, blood group $B$ was associated with lower merozoite invasion rates compared to blood group $\mathrm{O}$ in the heterozygous HbSC RBCs but not in the homozygous HbSS cells in vitro. This observation suggests that the anti-malaria protection conferred by RBC variants is possibly dependent on the interaction of multiple conditions.

\section{Conclusions}

Parasite invasion rate into erythrocytes is dependent on both erythrocyte blood group antigen and haemoglobin genotype, as blood group $\mathrm{O}$ and $\mathrm{B}$ provided protection via reduced merozoite invasion in RBCs containing $\mathrm{HbSS}$ relative to HbSC. Regardless of haemoglobin type, greater than $70 \%$ malaria patients had circulating ring stage parasites that differentiated into stage II gametocytes in 4 days.

\section{Supplementary information}

Supplementary information accompanies this paper at https://doi. org/10.1186/s12936-020-03275-9.

Additional file 1: Table S1. Results of in vitro merozoite invasion rates, asexual parasite multiplication rate and gametocyte conversion of ex vivo cultures.

\section{Abbreviations}

RBC: Red blood cell; Hb: Haemoglobin gene (beta globulin gene); HbS: Haemoglobin S; HbC: Haemoglobin C; G6PDd: Glucose 6 phosphate dehydrogenase deficiency; CPM: Complete parasite media; iCPM: Incomplete parasite media; NAG: N-acetyl glucosamine; PD: Parasite density.

\section{Acknowledgements \\ The authors are grateful to all the study participants as well as the staff of the Sickle cell laboratory, Korle-Bu Teaching Hospital as well as the Ewim poly- clinic. The authors also thank Dr Yaw Aniweh of WACCBIP, UG and Augustina Frimpong of the Immunology Department, NMIMR for critical review of this manuscript.}

\section{Authors' contributions}

LEA, KW, FS and GAA designed the study; LEA, PBN, RAT, FS, KW and FKA wrote the manuscript; DD, FKA, EBC, RAT and PBN performed the experiments; FKA and DD collected the samples. All authors read and approved the final manuscript.

\section{Funding}

This project was supported in part by the West African Centre for Cell Biology of Infectious Pathogens (WACCBIP) (ACE02-WACCBIP) and NIH Grant \# Al069314. The funders had no influence on the implementation of the project.

\section{Availability of data and materials}

All data generated or analysed during this study are included in this published article [and its additional information files].

\section{Ethics approval and consent to participate}

Ethical approval for the study was obtained from the Institutional Review Board of the Noguchi Memorial Institute for Medical Research (protocol \# 085/12-13 and \#024/14-15).

\section{Consent for publication}

Not applicable.

\section{Competing interests}

The authors declare that they have no competing interests.

\section{Author details}

${ }^{1}$ Department of Immunology, Noguchi Memorial Institute for Medical Research, University of Ghana, Accra, Ghana. ${ }^{2}$ West African Centre for Cell Biology of Infectious Pathogens, University of Ghana, Accra, Ghana. ${ }^{3}$ Ghana Institute of Clinical Genetics, Korle-Bu, Accra, Ghana. ${ }^{4}$ Microbiology and Immunology Department, Uniformed Services University of the Health Sciences, Bethesda, MD, USA.

Received: 25 January 2020 Accepted: 29 May 2020

Published online: 05 June 2020

\section{References}

1. Ruwende C, Khoo SC, Snow RW, Yates SN, Kwiatkowski D, Gupta S, et al. Natural selection of hemi- and heterozygotes for G6PD deficiency in Africa by resistance to severe malaria. Nature. 1995;376:246-9.

2. Bunyaratvej A, Butthep P, Sae-Ung N, Funcaroen S, Yuthavong Y. Reduced deformability of thalassemic erythrocytes and erythrocytes with abnormal hemoglobins and relation with susceptibility to Plasmodium falciparum invasion. Blood. 1992;79:2460-3. 
3. Friedman MJ. Erythrocytic mechanism of sickle cell resistance to malaria. Proc Natl Acad Sci USA. 1978;75:1994-7.

4. Agarwal A, Guindo A, Cissoko Y, Taylor JG, Coulibaly D, Kone A, et al. Hemoglobin $C$ associated with protection from severe malaria in the Dogon of Mali, a West African population with a low prevalence of hemoglobin S. Blood. 2000;96:2358-63.

5. Modiano D, Luoni G, Sirima BS, Simpore J, Verra F, Konate A, et al, Haemoglobin C protects against clinical Plasmodium falciparum malaria. Nature. 2001:414:305-8.

6. Gupta M, Chowdhuri AN. Relationship between ABO blood groups and malaria. Bull World Health Organ. 1980;58:913-5.

7. Taylor SM, Parobek CM, Fairhurst RM. Haemoglobinopathies and the clinical epidemiology of malaria: a systematic review and meta-analysis. Lancet Infect Dis. 2012;12:457-68.

8. Platt OS, Brambilla DJ, Rosse WF, Milner PF, Castro O, Steinberg MH, et al. Mortality in sickle cell disease. Life expectancy and risk factors for early death. N Engl J Med. 1994;330:1639-44.

9. May J, Evans JA, Timmann C, Ehmen C, Busch W, Thye T, et al. Hemoglobin variants and disease manifestations in severe falciparum malaria. JAMA. 2007;297:2220-6

10. Williams TN, Mwangi TW, Roberts DJ, Alexander ND, Weatherall DJ, Wambua S, et al. An immune basis for malaria protection by the sickle cell trait. PLoS Med. 2005;2:e128.

11. Williams TN, Mwangi TW, Wambua S, Alexander ND, Kortok M, Snow RW, et al. Sickle cell trait and the risk of Plasmodium falciparum malaria and other childhood diseases. J Infect Dis. 2005;192:178-86.

12. Gong L, Parikh S, Rosenthal PJ, Greenhouse B. Biochemical and immunological mechanisms by which sickle cell trait protects against malaria. Malar J. 2013;12:317

13. Cholera R, Brittain NJ, Gillrie MR, Lopera-Mesa TM, Diakite SA, Arie T, et al. Impaired cytoadherence of Plasmodium falciparum-infected erythrocytes containing sickle hemoglobin. Proc Natl Acad Sci USA. 2008;105:991-6.

14. Carlson J, Helmby H, Hill AV, Brewster D, Greenwood BM, Wahlgren M. Human cerebral malaria: association with erythrocyte rosetting and lack of anti-rosetting antibodies. Lancet. 1990;336:1457-60.

15. Lamonte G, Philip N, Reardon J, Lacsina JR, Majoros W, Chapman L, et al. Translocation of sickle cell erythrocyte microRNAs into Plasmodium falciparum inhibits parasite translation and contributes to malaria resistance. Cell Host Microbe. 2012;12:187-99.

16. Cyrklaff M, Sanchez CP, Kilian N, Bisseye C, Simpore J, Frischknecht F, et al. Hemoglobins $\mathrm{S}$ and $\mathrm{C}$ interfere with actin remodeling in Plasmodium falciparum-infected erythrocytes. Science. 2011:334:1283-6.

17. Ferreira A, Marguti I, Bechmann I, Jeney V, Chora A, Palha NR, et al. Sickle hemoglobin confers tolerance to Plasmodium infection. Cell. 2011;145:398-409.

18. Taylor SM, Fairhurst RM. Malaria parasites and red cell variants: when a house is not a home. Curr Opin Hematol. 2014;21:193-200.

19. Iyer J, Grüner AC, Rénia L, Snounou G, Preiser PR. Invasion of host cells by malaria parasites: a tale of two protein families. Mol Microbiol. 2007;65:231-49.

20. Dvorak JA, Miller LH, Whitehouse WC, Shiroishi T. Invasion of erythrocytes by malaria merozoites. Science. 1975;187:748-50.

21. Glushakova S, Yin D, Li T, Zimmerberg J. Membrane transformation during malaria parasite release from human red blood cells. Curr Biol. 2005:15:1645-50.

22. Bergmann-Leitner ES, Duncan EH, Mullen GE, Burge JR, Khan F, Long CA, et al. Critical evaluation of different methods for measuring the functional activity of antibodies against malaria blood stage antigens. Am J Trop Med Hyg. 2006;75:437-42.

23. Riglar DT, Richard D, Wilson DW, Boyle MJ, Dekiwadia C, Turnbull L, et al. Super-resolution dissection of coordinated events during malaria parasite invasion of the human erythrocyte. Cell Host Microbe. 2011;9:9-20.

24. Boyle MJ, Wilson DW, Richards JS, Riglar DT, Tetteh KK, Conway DJ, et al. Isolation of viable Plasmodium falciparum merozoites to define erythrocyte invasion events and advance vaccine and drug development. Proc Natl Acad Sci USA. 2010;107:14378-83.

25. Mensah-Brown HE, Amoako N, Abugri J, Stewart LB, Agongo G, Dickson EK, et al. Analysis of erythrocyte invasion mechanisms of Plasmodium falciparum clinical isolates across 3 malaria-endemic areas in Ghana. J Infect Dis. 2015;212:1288-97.
26. Theron M, Hesketh RL, Subramanian S, Rayner JC. An adaptable twocolor flow cytometric assay to quantitate the invasion of erythrocytes by Plasmodium falciparum parasites. Cytom Part A. 2010;77:1067-74.

27. Awandare GA, Nyarko PB, Aniweh Y, Ayivor-Djanie R, Stoute JA. Plasmodium falciparum strains spontaneously switch invasion phenotype in suspension culture. Sci Rep. 2018;8:5782.

28. Bruce MC, Alano P, Duthie S, Carter R. Commitment of the malaria parasite Plasmodium falciparum to sexual and asexual development. Parasitology. 1990;100:191-200.

29. Bancells C, Llora-Batlle O, Poran A, Notzel C, Rovira-Graells N, Elemento $\mathrm{O}$, et al. Revisiting the initial steps of sexual development in the malaria parasite Plasmodium falciparum. Nat Microbiol. 2019;4:144-54.

30. Buchholz K, Burke TA, Williamson KC, Wiegand RC, Wirth DF, Marti M. A high-throughput screen targeting malaria transmission stages opens new avenues for drug development. J Infect Dis. 2011;203:1445-53.

31. Boyle MJ, Wilson DW, Beeson JG. New approaches to studying Plasmodium falciparum merozoite invasion and insights into invasion biology. Int J Parasitol. 2013:43:1-10.

32. Mayer DC, Cofie J, Jiang L, Hartl DL, Tracy E, Kabat J, et al. Glycophorin $B$ is the erythrocyte receptor of Plasmodium falciparum erythrocytebinding ligand, EBL-1. Proc Natl Acad Sci USA. 2009;106:5348-52.

33. Bousema T, Drakeley C. Epidemiology and infectivity of Plasmodium falciparum and Plasmodium vivax gametocytes in relation to malaria control and elimination. Clin Microbiol Rev. 2011;24:377-410.

34. Joice R, Nilsson SK, Montgomery J, Dankwa S, Egan E, Morahan B, et al. Plasmodium falciparum transmission stages accumulate in the human bone marrow. Sci Transl Med. 2014;6:244re5.

35. Nacher M, Singhasivanon P, Silachamroon U, Treeprasertsuk S, Tosukhowong T, Vannaphan S, et al. Decreased hemoglobin concentrations, hyperparasitemia, and severe malaria are associated with increased Plasmodium falciparum gametocyte carriage. J Parasitol. 2002;88:97-101.

36. Gouagna LC, Bancone G, Yao F, Yameogo B, Dabire KR, Costantini C, et al. Genetic variation in human HBB is associated with Plasmodium falciparum transmission. Nat Genet. 2010;42:328-31.

37. Usui M, Prajapati SK, Ayanful-Torgby R, Acquah FK, Cudjoe E, Kakaney C, et al. Plasmodium falciparum sexual differentiation in malaria patients is associated with host factors and GDV1-dependent genes. Nat Commun. 2019;10:2140.

38. Rabinovich RN, Drakeley C, Djimde AA, Hall BF, Hay SI, Hemingway J, et al. malERA: an updated research agenda for malaria elimination and eradication. PLoS Med. 2017:14:e1002456.

39. Danquah I, Ziniel P, Eggelte TA, Ehrhardt S, Mockenhaupt FP. Influence of haemoglobins $S$ and $C$ on predominantly asymptomatic Plasmodium infections in northern Ghana. Trans R Soc Trop Med Hyg. 2010;104:713-9.

40. Amoah LE, Kakaney C, Kwansa-Bentum B, Kusi KA. Activity of herbal medicines on Plasmodium falciparum gametocytes: implications for malaria transmission in Ghana. PLoS ONE. 2015;10:e0142587.

41. Lambros C, Vanderberg JP. Synchronization of Plasmodium falciparum erythrocytic stages in culture. J Parasitol. 1979;65:418-20.

42. WHO. Microscopy for the detection, identification and quantification of malaria parasites on stained thick and thin blood films in research settings (version 1.0): methods manual. Geneva: World Health Organization; 2015.

43. WHO. Giemsa staining of malaria blood films. In: Malaria microscopy standard operating procedure. Manilla: World Health Organization Regional Office for the Western Pacific; 2016.

44. WHO. Malaria parasite counting. In: Malaria microscopy standard operating procedure. Manilla: World Health Organization Regional Office for the Western Pacific; 2016

45. Aidoo M, Terlouw DJ, Kolczak MS, McElroy PD, ter Kuile FO, Kariuki S, et al. Protective effects of the sickle cell gene against malaria morbidity and mortality. Lancet. 2002;359:1311-2.

46. Allison AC. Protection afforded by sickle-cell trait against subtertian malareal infection. BMJ. 1954;1:290-4.

47. Goncalves BP, Gupta S, Penman BS. Sickle haemoglobin, haemoglobin C and malaria mortality feedbacks. Malar J. 2016;15:26.

48. Mangano VD, Kabore Y, Bougouma EC, Verra F, Sepulveda N, Bisseye $C$, et al. Novel insights into the protective role of hemoglobin $\mathrm{S}$ and $\mathrm{C}$ against Plasmodium falciparum parasitemia. J Infect Dis. 2015;212:626-34. 
49. Mockenhaupt FP, Ehrhardt S, Cramer JP, Otchwemah RN, Anemana SD, Goltz K, et al. Hemoglobin C and resistance to severe malaria in Ghanaian children. J Infect Dis. 2004;190:1006-9.

50. Robert V, Read AF, Essong J, Tchuinkam T, Mulder B, Verhave JP, et al. Effect of gametocyte sex ratio on infectivity of Plasmodium falciparum to Anopheles gambiae. Trans R Soc Trop Med Hyg. 1996;90:621-4.

51. Goncalves BP, Kapulu MC, Sawa P, Guelbeogo WM, Tiono AB, Grignard $L$, et al. Examining the human infectious reservoir for Plasmodium falciparum malaria in areas of differing transmission intensity. Nat Commun. 2017;8:1133.

52. Nguitragool W, Mueller I, Kumpitak C, Saeseu T, Bantuchai S, Yorsaeng R, et al. Very high carriage of gametocytes in asymptomatic low-density Plasmodium falciparum and P. vivax infections in western Thailand. Parasites Vectors. 2017;10:512.
53. Omondi P, Burugu M, Matoke-Muhia D, Too E, Nambati EA, Chege W, et al. Gametocyte clearance in children, from western Kenya, with uncomplicated Plasmodium falciparum malaria after artemether-lumefantrine or dihydroartemisinin-piperaquine treatment. Malar J. 2019;18:398.

54. Olson JA, Nagel RL. Synchronized cultures of P. falciparum in abnormal red cells: the mechanism of the inhibition of growth in $\mathrm{HbCC}$ cells. Blood. 1986;67:997-1001.

\section{Publisher's Note}

Springer Nature remains neutral with regard to jurisdictional claims in published maps and institutional affiliations.
Ready to submit your research? Choose BMC and benefit from:

- fast, convenient online submission

- thorough peer review by experienced researchers in your field

- rapid publication on acceptance

- support for research data, including large and complex data types

- gold Open Access which fosters wider collaboration and increased citations

- maximum visibility for your research: over 100M website views per year

At BMC, research is always in progress.

Learn more biomedcentral.com/submissions 